\title{
CONGRUENCES BETWEEN HILBERT MODULAR FORMS: CONSTRUCTING ORDINARY LIFTS, II
}

\author{
Thomas Barnet-Lamb, Toby Gee and David Geraghty
}

\begin{abstract}
In this paper, we improve on the results of our earlier paper [BLGG12], proving a near-optimal theorem on the existence of ordinary lifts of a mod $l$ Hilbert modular form for any odd prime $l$.
\end{abstract}

\section{CONTENTS}

1. Introduction $\quad 67$

2. The adequate case 68

$\begin{array}{ll}3 . & \text { Inadequate cases }\end{array}$

$\begin{array}{ll}\text { Acknowledgment } & 71\end{array}$

$\begin{array}{ll}\text { References } & 71\end{array}$

\section{Introduction}

Let $F$ be a totally real field with absolute Galois group $G_{F}$, and let $l$ be an odd prime number. In our earlier paper [BLGG12], we proved a general result on the existence of ordinary modular lifts of a given modular representation $\bar{\rho}: G_{F} \rightarrow \mathrm{GL}_{2}\left(\overline{\mathbb{F}}_{l}\right)$; we refer the reader to the introduction of $o p$. cit. for a detailed discussion of the problem of constructing such a lift, and of our techniques for doing so.

The purpose of this paper is to improve on the hypotheses imposed on $\bar{\rho}$, removing some awkward assumptions on its image; in particular, if $l=3$ then the results of [BLGG12] were limited to some cases where $\bar{\rho}$ was induced from a quadratic character, whereas our main theorem is the following.

Theorem A. Suppose that $l>2$ is prime, that $F$ is a totally real field, and that $\bar{\rho}: G_{F} \rightarrow \mathrm{GL}_{2}\left(\overline{\mathbb{F}}_{l}\right)$ is irreducible and modular. Assume that $\left.\bar{\rho}\right|_{G_{F_{v}}}$ is reducible at all places $v \mid l$ of $F$.

If $l=5$ and the projective image of $\left.\bar{\rho}\right|_{G_{F\left(\zeta_{5}\right)}}$ is isomorphic to $\mathrm{PSL}_{2}\left(\mathbb{F}_{5}\right)$, assume further that there is a finite solvable totally real extension $F^{\prime} / F$ such that $\left.\bar{\rho}\right|_{G_{F^{\prime}}}$ is conjugate to a representation valued in $\mathrm{GL}_{2}\left(\mathbb{F}_{5}\right)$.

Then $\bar{\rho}$ has a modular lift $\rho: G_{F} \rightarrow \mathrm{GL}_{2}\left(\overline{\mathbb{Q}}_{l}\right)$, which is ordinary at all places $v \mid l$.

Received by the editors December 21, 2012.

2000 Mathematics Subject Classification. 11F33. 
(Note that the assumption that $\left.\bar{\rho}\right|_{G_{F v}}$ is reducible at all places $v \mid l$ of $F$ is necessary.) Our methods are based on those of [BLGG12]. The reason that we are now able to prove a stronger result is that the automorphy lifting results that we employed in [BLGG12] have since been optimized in [BLGGT10] and [Tho12]; in particular, we make extensive use of the results of the appendix to [BLGG13], which improves on a lifting result of [BLGGT10], and classifies the subgroups of $\mathrm{GL}_{2}\left(\overline{\mathbb{F}}_{l}\right)$, which are adequate in the sense of [Tho12]. In Section 2, we use these results to prove Theorem A, except in the case that $l=3$ or 5 and the projective image of $\bar{\rho}\left(G_{F\left(\zeta_{l}\right)}\right)$ is isomorphic to $\mathrm{PSL}_{2}\left(\mathbb{F}_{l}\right)$, and certain cases where $\bar{\rho}$ is dihedral. In the dihedral cases, the result is proved in [All12]. In the remaining cases, the adequacy hypothesis we require fails, but in Section 3 we handle this case completely when $l=3$ by making use of the Langlands-Tunnell theorem, and we prove a partial result when $l=5$ using the results of [SBT97].

1.1. Notation. If $M$ is a field, we let $G_{M}$ denote its absolute Galois group. We write $\bar{\varepsilon}$ for the mod $l$ cyclotomic character. We fix an algebraic closure $\overline{\mathbb{Q}}$ of $\mathbb{Q}$, and regard all algebraic extensions of $\mathbb{Q}$ as subfields of $\overline{\mathbb{Q}}$. For each prime $p$ we fix an algebraic closure $\overline{\mathbb{Q}}_{p}$ of $\mathbb{Q}_{p}$, and we fix an embedding $\overline{\mathbb{Q}} \hookrightarrow \overline{\mathbb{Q}}_{p}$. In this way, if $v$ is a finite place of a number field $F$, we have a homomorphism $G_{F_{v}} \hookrightarrow G_{F}$. We also fix an embedding $\overline{\mathbb{Q}} \hookrightarrow \mathbb{C}$.

We normalize the definition of Hodge-Tate weights so that all the Hodge-Tate weights of the $l$-adic cyclotomic character $\varepsilon$ are -1 . We refer to a two-dimensional potentially crystalline representation with all pairs of labelled Hodge-Tate weights equal to $\{0,1\}$ as a weight 0 representation. (The reason for this terminology is that the Galois representations associated to an automorphic representation, which is cohomological of weight 0 have these Hodge-Tate weights.)

If $F$ is a totally real field, then a continuous representation $\bar{r}: G_{F} \rightarrow \mathrm{GL}_{2}\left(\overline{\mathbb{F}}_{l}\right)$ is said to be modular if there exists a regular algebraic automorphic representation $\pi$ of $\mathrm{GL}_{2}\left(\mathbb{A}_{F}\right)$, such that $\bar{r}_{l}(\pi) \cong \bar{r}$, where $r_{l}(\pi)$ is the $l$-adic Galois representation associated with $\pi$.

We let $\zeta_{l}$ be a primitive $l$ th root of unity.

\section{The adequate case}

2.1. The notion of an adequate subgroup of $\mathrm{GL}_{n}\left(\overline{\mathbb{F}}_{l}\right)$ is defined in [Tho12]. We will not need to make use of the actual definition; instead, we will use the following classification result. Note that by definition an adequate subgroup of $\mathrm{GL}_{n}\left(\overline{\mathbb{F}}_{l}\right)$ necessarily acts irreducibly on $\overline{\mathbb{F}}_{l}^{n}$.

Proposition 2.1.1. Suppose that $l>2$ is a prime, and that $G$ is a finite subgroup of $\mathrm{GL}_{2}\left(\overline{\mathbb{F}}_{l}\right)$, which acts irreducibly on $\overline{\mathbb{F}}_{l}^{2}$. Then precisely one of the following is true:

- We have $l=3$, and the image of $G$ in $\mathrm{PGL}_{2}\left(\overline{\mathbb{F}}_{3}\right)$ is conjugate to $\mathrm{PSL}_{2}\left(\mathbb{F}_{3}\right)$.

- We have $l=5$, and the image of $G$ in $\mathrm{PGL}_{2}\left(\overline{\mathbb{F}}_{5}\right)$ is conjugate to $\mathrm{PSL}_{2}\left(\mathbb{F}_{5}\right)$.

- $G$ is adequate.

Proof. This is Proposition A.2.1 of [BLGG13]. 
In the case that $\bar{\rho}\left(G_{F\left(\zeta_{l}\right)}\right)$ is adequate, our main result follows exactly as in section 6 of [BLGG12], using the results of Appendix A of [BLGG13] (which in turn build on the results of [BLGGT10]). We obtain the following theorem.

Theorem 2.1.2. Suppose that $l>2$ is prime, that $F$ is a totally real field, and that $\bar{\rho}: G_{F} \rightarrow \mathrm{GL}_{2}\left(\overline{\mathbb{F}}_{l}\right)$ is irreducible and modular. Suppose also that $\bar{\rho}\left(G_{F\left(\zeta_{l}\right)}\right)$ is adequate. Then:

(1) There is a finite solvable extension of totally real fields $L / F$ which is linearly disjoint from $\bar{F}^{\operatorname{ker} \bar{\rho}}$ over $F$, such that $\left.\bar{\rho}\right|_{G_{L}}$ has a modular lift $\rho_{L}: G_{L} \rightarrow$ $\mathrm{GL}_{2}\left(\overline{\mathbb{Q}}_{l}\right)$ of weight 0 , which is ordinary at all places $v \mid l$.

(2) If furthermore $\left.\bar{\rho}\right|_{G_{F_{v}}}$ is reducible at all places $v \mid l$, then $\bar{\rho}$ itself has a modular lift $\rho: G_{F} \rightarrow \mathrm{GL}_{2}\left(\overline{\mathbb{Q}}_{l}\right)$ of weight 0 , which is ordinary at all places $v \mid l$.

Proof. First, note that (2) is easily deduced from (1) using the results of Section 3 of [Gee11] (which build on Kisin's reinterpretation of the Khare-Wintenberger method). Indeed, the proofs of Theorems 6.1.5 and 6.1.7 of [BLGG12] go through unchanged in this case.

Similarly, (1) is easily proved in the same way as Proposition 6.1.3 of [BLGG12] (and in fact the proof is much shorter). First, note that the proof of Lemma 6.1.1 of [BLGG12] goes through unchanged to show that there is a finite solvable extension of totally real fields $L / F$ which is linearly disjoint from $\bar{F}^{\operatorname{ker} \bar{\rho}}$ over $F$, such that $\left.\bar{\rho}\right|_{G_{L}}$ has a modular lift $\rho^{\prime}: G_{L} \rightarrow \mathrm{GL}_{2}\left(\overline{\mathbb{Q}}_{l}\right)$ of weight 0 which is potentially crystalline at all places dividing $l$, and in addition both $\left.\bar{\rho}\right|_{G_{L_{w}}}$ and $\left.\bar{\varepsilon}\right|_{G_{L_{w}}}$ are trivial for each place $w \mid l$ (and in particular, $\left.\bar{\rho}\right|_{G_{L_{w}}}$ admits an ordinary lift of weight 0 ), and $\bar{\rho}$ is unramified at all finite places. By Lemma 4.4.1 of [GK12], $\left.\rho^{\prime}\right|_{G_{L_{w}}}$ is potentially diagonalizable in the sense of [BLGGT10] for all places $w \mid l$ of $L$.

Choose a CM quadratic extension $M / L$ that is linearly disjoint from $L\left(\zeta_{l}\right)$ over $L$, in which all places of $L$ dividing $l$ split. We can now apply Theorem A.4.1 of [BLGG13] (with $F^{\prime}=F=M, S$ the set of places of $L$ dividing $l$, and $\rho_{v}$ an ordinary lift of $\left.\bar{\rho}\right|_{G_{L_{w}}}$ for each $w \mid l)$ to see that $\left.\bar{\rho}\right|_{G_{M}}$ has an ordinary automorphic lift $\rho_{M}: G_{M} \rightarrow \mathrm{GL}_{2}\left(\overline{\mathbb{Q}}_{l}\right)$ of weight 0 .

The argument of the last paragraph of the proof of Proposition 6.1.3 of [BLGG12] (which uses the Khare-Wintenberger method to compare deformation rings for $\left.\bar{\rho}\right|_{G_{L}}$ and $\left.\bar{\rho}\right|_{G_{M}}$ ) now goes over unchanged to complete the proof.

\section{Inadequate cases}

3.1. The first inadequate case. We now consider the case that $l=3$ and $\left.\bar{\rho}\right|_{G_{F\left(\zeta_{3}\right)}}$ is irreducible, but $\bar{\rho}\left(G_{F\left(\zeta_{3}\right)}\right)$ is not adequate. By Proposition 2.1.1, this means that the projective image of $\bar{\rho}\left(G_{F\left(\zeta_{3}\right)}\right)$ is isomorphic to $\mathrm{PSL}_{2}\left(\mathbb{F}_{3}\right)$, and is in particular solvable. We now use the Langlands-Tunnell theorem to prove our main theorem in this case.

Theorem 3.1.1. Suppose that $F$ is a totally real field, and that $\bar{\rho}: G_{F} \rightarrow \mathrm{GL}_{2}\left(\overline{\mathbb{F}}_{3}\right)$ is irreducible and modular. Assume that $\left.\bar{\rho}\right|_{G_{F_{v}}}$ is reducible at all places $v \mid 3$ of $F$, and that the projective image of $\bar{\rho}\left(G_{F\left(\zeta_{3}\right)}\right)$ is isomorphic to $\mathrm{PSL}_{2}\left(\mathbb{F}_{3}\right)$.

Then $\bar{\rho}$ has a modular lift $\rho: G_{F} \rightarrow \mathrm{GL}_{2}\left(\overline{\mathbb{Q}}_{3}\right)$ which is ordinary at all places $v \mid 3$. 
Proof. First, note that since the projective image of $\bar{\rho}\left(G_{F\left(\zeta_{3}\right)}\right)$ is isomorphic to $\mathrm{PSL}_{2}\left(\mathbb{F}_{3}\right)$, the projective image of $\bar{\rho}$ itself is isomorphic to $\mathrm{PSL}_{2}\left(\mathbb{F}_{3}\right)$ or $\mathrm{PGL}_{2}\left(\mathbb{F}_{3}\right)$ (see, for example, Theorem 2.47(b) of [DDT97]).

Choose a finite solvable extension of totally real fields $L / F$ which is linearly disjoint from $\bar{F}^{\operatorname{ker} \bar{\rho}}$ over $F$, with the further property that $\left.\bar{\rho}\right|_{G_{L_{w}}}$ is unramified for each place $w \mid l$ of $L$. Exactly as in the proof of Theorem 2.1.2, by the results of Section 3 of [Gee11] it suffices to show that $\left.\bar{\rho}\right|_{G_{L}}$ has a modular lift of weight 0 , which is potentially crystalline at each place $w \mid l$. By Hida theory, it in fact suffices to find some ordinary modular lift of $\left.\bar{\rho}\right|_{G_{L}}$ (not necessarily of weight 0 ).

Since the projective image of $\bar{\rho}$ is isomorphic to $\mathrm{PSL}_{2}\left(\mathbb{F}_{3}\right)$ or $\mathrm{PGL}_{2}\left(\mathbb{F}_{3}\right)$, the image of $\bar{\rho}$ is contained in $\overline{\mathbb{F}}_{3}^{\times} \mathrm{GL}_{2}\left(\mathbb{F}_{3}\right)$. Then the Langlands-Tunnell theorem implies that $\left.\bar{\rho}\right|_{G_{L}}$ has a modular lift $\rho$ corresponding to a Hilbert modular form of parallel weight one. This follows from the discussion after Theorem 5.1 of [Wil95] which also shows that the natural map $\rho\left(G_{L}\right) \rightarrow \bar{\rho}\left(G_{L}\right)$ may be assumed to be an isomorphism. Since $\left.\bar{\rho}\right|_{G_{L_{w}}}$ is unramified at each place $w \mid l$ of $L$, this implies that $\rho$ is ordinary, as required.

3.2. The second inadequate case. We now suppose that $l=5$, that $\left.\bar{\rho}\right|_{G_{F\left(\zeta_{5}\right)}}$ is irreducible but its image is not adequate. Then $\bar{\rho}\left(G_{F\left(\zeta_{5}\right)}\right)$ has projective image conjugate to $\mathrm{PSL}_{2}\left(\mathbb{F}_{5}\right)$, and we see that $\bar{\rho}\left(G_{F}\right)$ has projective image conjugate to either $\mathrm{PGL}_{2}\left(\mathbb{F}_{5}\right)$ or $\mathrm{PSL}_{2}\left(\mathbb{F}_{5}\right)$. (This follows from [DDT97, Prop. 2.47].) Thus, after conjugating, we may assume that $\bar{\rho}: G_{F} \rightarrow \mathrm{GL}_{2}\left(\overline{\mathbb{F}}_{5}\right)$ takes values in $\overline{\mathbb{F}}_{5}^{\times} \mathrm{GL}_{2}\left(\mathbb{F}_{5}\right)$.

In order to apply the results of [SBT97], we need to assume further that there is a finite solvable totally real extension $F^{\prime} / F$ such that $\left.\bar{\rho}\right|_{G_{F^{\prime}}}$ is valued in $\mathrm{GL}_{2}\left(\mathbb{F}_{5}\right)$. (This condition is not automatic, but it holds if the projective image of $\bar{\rho}\left(G_{F}\right)$ is isomorphic to $\mathrm{PSL}_{2}\left(\mathbb{F}_{5}\right)$.)

Theorem 3.2.1. Suppose that $F$ is a totally real field, and that $\bar{\rho}: G_{F} \rightarrow \mathrm{GL}_{2}\left(\overline{\mathbb{F}}_{5}\right)$ is irreducible and modular. Assume that $\left.\bar{\rho}\right|_{G_{F v}}$ is reducible at all places $v \mid 5$ of $F$, and that the projective image of $\bar{\rho}\left(G_{F\left(\zeta_{5}\right)}\right)$ is isomorphic to $\mathrm{PSL}_{2}\left(\mathbb{F}_{5}\right)$. Assume further that there is a finite solvable totally real extension $F^{\prime} / F$ so that $\left.\bar{\rho}\right|_{G_{F^{\prime}}}$ is conjugate to a representation valued in $\mathrm{GL}_{2}\left(\mathbb{F}_{5}\right)$.

Then $\bar{\rho}$ has a modular lift $\rho: G_{F} \rightarrow \mathrm{GL}_{2}\left(\overline{\mathbb{Q}}_{5}\right)$ which is ordinary at all places $v \mid 5$.

Proof. Since $\bar{\rho}$ is totally odd, we can replace $F^{\prime} / F$ by a further finite solvable totally real extension and assume that $\left.\bar{\rho}\right|_{G_{F^{\prime}}}$ takes values in $\mathrm{GL}_{2}\left(\mathbb{F}_{5}\right)$ and has determinant equal to the cyclotomic character. Now, as in the proof of Theorem 2.1.2, to prove the current theorem, it suffices to show that $\left.\bar{\rho}\right|_{G_{F^{\prime}}}$ has a modular lift of weight 0 , which is ordinary at each $v \mid 5$. (The only thing that needs to be checked is that Proposition 3.1 .5 of [Gee11] applies to $\left.\bar{\rho}\right|_{G_{F^{\prime}}}$. The only hypothesis which is not immediate is that if the projective image of $\left.\bar{\rho}\right|_{G_{F^{\prime}}}$ is $\mathrm{PGL}_{2}\left(\mathbb{F}_{5}\right)$, then $\left[F^{\prime}\left(\zeta_{5}\right): F^{\prime}\right]=4$. To see this, note that if $\left[F^{\prime}\left(\zeta_{5}\right): F^{\prime}\right]=2$, then since the determinant of $\left.\bar{\rho}\right|_{G_{F^{\prime}}}$ is the $\bmod 5$ cyclotomic character, it has image $\{ \pm 1\}$. This implies that the projective image is $\operatorname{PSL}_{2}\left(\mathbb{F}_{5}\right)$, as required.)

By [SBT97, Theorem 1.2], there exists an elliptic curve $E / F^{\prime}$ such that $\left.E[5] \cong \bar{\rho}\right|_{G_{F^{\prime}}}$ and the image of $G_{F^{\prime}}$ in $\operatorname{Aut}(E[3])$ contains $\mathrm{SL}_{2}\left(\mathbb{F}_{3}\right)$ (and hence its image is equal to $\operatorname{Aut}(E[3])$ since the determinant is totally odd). We may further suppose that $E$ has good ordinary reduction at each prime of $F^{\prime}$ dividing 5 . (To see this, note that we may 
incorporate Ekedahl's effective version of the Hilbert Irreducibility Theorem [Eke90] into the proof of [SBT97, Theorem 1.2] exactly as is done in [Tay03, Lemma 2.3].) By the Langlands-Tunnell theorem, $E[3]$ has a modular lift corresponding to a Hilbert modular form $f_{0}$ of parallel weight 1 . Replacing $F^{\prime}$ by a finite totally real solvable extension linearly disjoint from ${\overline{F^{\prime}}}^{\text {ker } E[3]}$, we may assume that $f_{0}$ is ordinary at each prime dividing 3. By Hida theory, $E[3]$ then has a modular lift corresponding to a Hilbert modular form of parallel weight 2 , which is ordinary at each prime dividing 3. Note that the conditions of the modularity lifting theorem [Gee09, Theorem 1.1], applied to $\rho:=T_{3} E$, are satisfied. (For the third condition, note that $\left.E[3]\right|_{G_{F^{\prime}\left(\zeta_{3}\right)}}$ is irreducible as $\left.E[3]\right|_{G_{F^{\prime}}}$ has non-dihedral image.) It follows that $T_{3} E$ is modular and hence that $T_{5} E$ is modular. Thus we have exhibited a modular lift of $\left.\bar{\rho}\right|_{G_{F^{\prime}}} \cong E[5]$ which has weight 0 and is ordinary at each prime above 5 .

Finally, we deduce our main result from Theorems 2.1.2, 3.1.1 and 3.2.1.

Proof of Theorem A. If $\left.\bar{\rho}\right|_{G_{F\left(\zeta_{l}\right)}}$ is reducible, then $\bar{\rho}$ is dihedral, and the result follows from Lemma 5.1 .2 of [All12]. If $l=3$ (respectively $l=5$ ) and the projective image of $\bar{\rho}\left(G_{F\left(\zeta_{l}\right)}\right)$ is isomorphic to $\mathrm{PSL}_{2}\left(\mathbb{F}_{l}\right)$, then the result follows from Theorem 3.1.1 (respectively, from Theorem 3.2.1). In all other cases, we see from Proposition 2.1.1 that $\bar{\rho}\left(G_{F\left(\zeta_{l}\right)}\right)$ is adequate and the result follows from Theorem 2.1.2(2).

\section{Acknowledgment}

We would like to thank Vincent Pilloni for pointing out to us that we could make use of the results of [SBT97].

\section{References}

[All12] P. Allen, Modularity of nearly ordinary 2-adic residually dihedral Galois representations, 2012.

[BLGG12] T. Barnet-Lamb, T. Gee and D. Geraghty, Congruences between Hilbert modular forms: constructing ordinary lifts, Duke Math. J. 161(8) (2012), 1521-1580.

[BLGG13] _, Serre weights for rank two unitary groups, Math. Ann., 2013, http://link.springer.com/article/10.1007/s00208-012-0893-y.

[BLGGT10] T. Barnet-Lamb, T. Gee, D. Geraghty and R. Taylor, Potential automorphy and change of weight, 2010, http://annals.math.princeton.edu/articles/7604

[DDT97] H. Darmon, F. Diamond and R. Taylor, Fermat's last theorem, Elliptic curves, modular forms \& Fermat's last theorem (Hong Kong, 1993), Int. Press, Cambridge, MA, 1997, 2-140.

[Eke90] T. Ekedahl, 'An effective version of Hilbert's irreducibility theorem', in Séminaire de Théorie des Nombres, Paris 1988-1989, Progr. Math., 91, Birkhäuser, Boston, MA, 1990, 241-249.

[Gee09] T. Gee, Erratum - a modularity lifting theorem for weight two Hilbert modular forms, Math. Res. Lett. 16(1) (2009), 57-58.

[Gee11] - Automorphic lifts of prescribed types, Math. Ann. 350(1) (2011), 107-144.

[GK12] T. Gee and M. Kisin, The Breuil-Mézard conjecture for potentially Barsotti-Tate representations, 2012.

[SBT97] N.I. Shepherd-Barron and R. Taylor, mod 2 and $\bmod 5$ icosahedral representations, J. Amer. Math. Soc. 10(2) (1997), 283-298.

[Tay03] R. Taylor, On icosahedral Artin representations. II, Amer. J. Math. 125(3) (2003), 549-566.

[Tho12] J. Thorne, On the automorphy of l-adic Galois representations with small residual image, J. Inst. Math. Jussieu 11(4) (2012), 855-920.

[Wil95] A. Wiles, Modular elliptic curves and Fermat's last theorem, Ann. Math. (2) 141(3) (1995), $443-551$. 
Department of Mathematics, Brandeis University, 415 South St, Waltham, MA 02453, USA

E-mail address: tbl@brandeis.edu

Department of Mathematics, Imperial College London, South Kensington Campus,, ExhiBITION RD, LONDON SW7 2AZ, UK

E-mail address: toby.gee@imperial.ac.uk

Princeton University and Institute for Advanced Study, 1 Einstein Dr, Princeton, NJ 08540, USA

E-mail address: geraghty@math.princeton.edu 